\title{
Aufsatz
}

Elbridge Colby*

\section{Deutschland am Scheidepunkt - eine aktive Verteidigungs- und Bündnispolitik ist überfällig}

https://doi.org/10.1515/sirius-2021-3003

Kurzfassung: Seit den 1990er Jahren bis in die jüngste Vergangenheit waren die USA und ihr Militär weitaus stärker als jeder andere Staat. Sie konnten mit jeder plausibel erscheinenden Bedrohung für ihr weltweites Bündnisnetzwerk nahezu aus eigener Kraft umgehen und planten für Szenarien, in denen es darum ging, gleichzeitig Angriffe auf mehrere Verbündete in verschiedenen Regionen der Welt abzuwehren. Der Aufstieg Chinas zur Supermacht hat die amerikanische und damit transatlantische Verteidigungsplanung jedoch grundlegend verändert. Heute müssen die USA mit einem Gegner rechnen, der sie militärisch mit einer gewissen Wahrscheinlichkeit in Asien, einem der wichtigsten Operationsgebiete der Welt, besiegen könnte. Bereits in der National Defense Strategie von 2018 sind sie daher von ihrem ,Zwei-Kriege-Planungsstandard“ gegen „Schurkenstaaten“ abgewichen, um sich auf das Gewinnen eines Krieges gegen eine Großmacht - nämlich China - zu konzentrieren. Gleichzeitig hat der Aufstieg Chinas nicht die Bedrohung beseitigt, die von Russland für die europäischen NATO-Staaten ausgeht. Diese Entwicklungen werden sehr unmittelbare, ja dramatische Folgen für die Sicherheit Europas haben: In einem Krieg mit Russland, müssten die USA ihr militärisches Engagement begrenzen, um in Asien nicht zu große Risiken einzugehen und dadurch opportunistischen Angriffen Chinas etwa gegen Taiwan Tür und Tor zu öffnen. Dies dürfte eine erhebliche und wachsende Lücke in Europas Verteidigung nach sich ziehen, die von den europäischen NATO-Staaten gefüllt werden muss. Deutschland kommt hierbei eine Schlüsselposition zu. Der Beitrag zeigt diese Zusammenhänge auf und arbeitet die besondere Rolle der Bundesrepublik heraus. Er kommt zu dem Schluss, dass Deutschland eine Führungsrolle bei der konventionelle Verteidigung Europas - insbesondere der östlichen NATO-Staaten und Skandinaviens - zu kommt. Sollten sich die europäischen Verbündeten, spe-

\footnotetext{
*Kontakt: Elbridge Colby ist Direktor bei der Marathon Initiative. In den Jahren 2017-2018 war er als leitender Beamter an der Entwicklung der National Defense Strategy 2018 des Pentagon beteiligt; E-Mail: elbridge.colby@themarathoninitiative.org
}

ziell Deutschland, weiterhin weigern, ihre Verteidigungsanstrengungen zu erhöhen, werden die USA das Sicherheitsvakuum in Europa kaum füllen, sondern eher die Risiken akzeptieren, die eine solche Verweigerung für die Sicherheit Europas mit sich bringt.

Schlüsselwörter: Deutschland, NATO, Verteidigungspolitik, europäische Sicherheit, asiatische Sicherheit

Abstract: From the 1990s until the recent past, the United States and thus its military was far more powerful than any other state. Washington could deal with any plausible threat to its global alliance network almost on its own, planning for scenarios that involved simultaneously fending off attacks on multiple allies in different regions of the world. The rise of China as a superpower has, however, fundamentally changed U.S., and thus transatlantic, defense planning. Today, the United States must reckon with an adversary that might well be able to defeat it in Asia, the world's key theater. Already in the 2018 National Defense Strategy, therefore, the United States has departed from its „two wars planning standard“ against „rogue states“ to focus on winning a war against one great power - namely China. At the same time, the rise of China has not eliminated the threat posed by Russia to European NATO countries. These developments will have very immediate, indeed dramatic, consequences for European security: In a war with Russia, the U.S. would have to limit its military involvement in Europe to avoid taking too great a risk in Asia and thereby opening the door to opportunistic attacks by China against Taiwan, for example. This is likely to result in a significant and growing gap in Europe's defense, which will have to be filled by European NATO countries. Germany has a key position in this regard. The article points out these connections and elaborates the special role of the Federal Republic. It concludes that Germany has a leading role to play in the conventional defense of Europe, especially the eastern NATO countries and Scandinavia. If European allies, especially Germany, continue to refuse to increase their defense efforts, the United States is unlikely to fill the security vacuum in 
Europe, but will rather accept the risks that such a refusal poses to European security.

Keywords: Germany, NATO, defence policy, European security, Asian security

\section{Einleitung}

Der Aufstieg Chinas hat die nach dem Kalten Krieg maßgeblichen Grundlagen der transatlantischen Verteidigungsplanung tiefgreifend verändert. Von den 1990er Jahren bis in die jüngste Vergangenheit hinein waren die Vereinigten Staaten - und folglich ihr Militär - weitaus stärker als jeder andere Staat. Außerdem waren die größten Volkswirtschaften der Welt überwiegend Verbündete der USA. Zumindest in militärischer Hinsicht deckten sich ihre Interessen weitgehend mit denen der USA. Da Russland einen steilen Absturz von seiner einstigen sowjetischen Größe durchmachte und China noch immer vergleichsweise unterentwickelt war, konzentrierte sich die US-Verteidigungsplanung in dieser Zeit hauptsächlich auf die Verteidigung der Verbündeten und Partner Washingtons gegen sogenannte „Schurkenstaaten“ wie Irak, Iran und Nordkorea.

In diesem geopolitisch günstigen Umfeld konnte das US-Militär bei vergleichsweise hohen Verteidigungsausgaben mit jeder plausibel erscheinenden Bedrohung des breitgespannten Bündnisnetzwerks weitgehend aus eigener Kraft fertigwerden. Mehr noch: Washington konnte darauf hoffen, dies gleichzeitig auf mehreren Kriegsschauplätzen zu tun. Man plante für Szenarien, in denen es darum ging, gleichzeitig Angriffe auf mehrere Verbündete abzuwehren - zum Beispiel einen Angriff Irans auf einen Partner am Persischen Golf und einen parallel erfolgenden Einmarsch nordkoreanischer Truppen in Südkorea. Dies war der vielgerühmte „Zwei-Kriege-Standard“ der US-amerikanischen Verteidigungsplanung. Tatsächlich war die militärische Überlegenheit der USA so groß, dass die USA in ihrer Verteidigungsplanung nicht nur die Verteidigung ihrer Bündnispartner garantierten, sondern auch „Regimewechsel“ anstreben konnten - den gewaltsamen Sturz der Regierung eines Feindes, an deren Stelle eine freundlicher gesinnte treten sollte.

Dies war eine ungewöhnlich sichere und bequeme Situation für die meisten US-Verbündeten. Die Vereinigten Staaten und ihr Militär verschafften ihren Verbündeten ein sehr hohes Maß an Sicherheit vor militärischen Bedrohungen. Washington war auch bereit, die Sicherheitsgarantien für seine Verbündeten öffentlich zu bestätigen. Die im Vergleich zu den anderen Bündnispartnern hohen
Verteidigungsausgaben waren in den USA kein Anlass für politische Opposition. Die Vereinigten Staaten besaßen mithin den Willen und die Fähigkeiten, um ihre Verbündeten gegen jede plausibel erscheinende militärische Bedrohung zu verteidigen. Mehr noch: Das US-Militär hatte bei den Fähigkeiten zu jener Art von Kriegsführung mit Expeditionsstreitkräften, auf die es in diesen Jahren anzukommen schien, einen so großen Vorsprung vor seinen Verbündeten, dass nicht einmal wirklich klar war, ob die Beiträge von Verbündeten einen großen Unterschied machen würden, auch wenn sie selbst dies wollten. Es war daher nicht weiter überraschend, dass viele US-Verbündete sich in diesen Jahren de facto demilitarisierten, insbesondere in West- und Mitteleuropa, wo es keine direkte militärische Bedrohung mehr zu geben schien. Nachdem die Sowjetunion keine militärische Bedrohung mehr für die Verbündeten der USA in Europa darstellte und sie auf die Fähigkeit und Entschlossenheit Washingtons vertrauen konnten, sie im Notfall zu verteidigen, konnten die Europäer ihre Verteidigungsausgaben verringern und die eingesparten Mittel in anderen Politikbereichen verwenden, wie Bildung, Sozialleistungen oder Umweltschutz und Klimapolitik.

Diese Entwicklung ließ sich ganz besonders in Deutschland beobachten. Die Bundesrepublik hatte während des Kalten Krieges eindrucksvolle Streitkräfte aufgebaut, aber diese wurden nach den Katastrophen, die das Land in der ersten Hälfte des 20. Jahrhunderts durchlebt hatte, immer misstrauisch beäugt. Das nunmehr wiedervereinigte Deutschland, das unter dem Einfluss von Ideen über das Ende der Geschichte in einem friedlichen europäischen Kontinent aufging, ergriff bereitwillig die Gelegenheit, um die robuste Bundeswehr aus der Zeit des Kalten Krieges funktional aufzulösen. ${ }^{1}$ In den 90er Jahren erzeugte diese faktische Demilitarisierung keine nennenswerten Spannungen mit den Vereinigten Staaten. Gelegentliche eindringliche Mahnungen von US-Verteidigungsministern bei NATO-Ministertagungen, die Verbündeten sollten mehr tun, hatten keine ernsten Verstimmungen, geschweige denn Brüche, in den transatlantischen Beziehungen zur Folge.

In einem sehr realen Sinne war die Demilitarisierung Deutschlands nach dem Kalten Krieg eine rationale Entscheidung. Deutschland war sicher, die deutsche Politik unterstützte keine aktive militärische Rolle, und diese politische Strategie schien keine wirklich gravierenden Probleme mit den Vereinigten Staaten oder den europäischen Partnern Berlins zu verursachen. Ein entmilitari-

1 Bagger 2019. 
siertes Deutschland passte recht gut in die kooperative Welt nach dem Ende des Kalten Krieges.

\section{Grundlegende Veränderungen im internationalen militärischen Gleichgewicht}

Aber diese Welt ist untergegangen, wie die amtierende deutsche Verteidigungsministerin $\mathrm{zu}$ Recht festgestellt hat. ${ }^{2}$ Und dies vor allem aus einem Grund: wegen des Aufstiegs Chinas, der mittlerweile unaufhaltsam zu sein scheint. China ist schon heute eine gigantische Volkswirtschaft - entweder die größte oder die zweitgrößte in der Welt, je nachdem, welche Kennzahlen man zugrunde legt. ${ }^{3}$ Und die chinesischen Streitkräfte, die Volksbefreiungsarmee (PLA), sind mittlerweile, mit Ausnahme derjenigen der Vereinigten Staaten, weltweit mit Abstand das schlagkräftigste konventionelle Militär. ${ }^{4}$ Tatsächlich stellt China die gewaltigste militärische Herausforderung für das USMilitär und seine Verbündeten seit den Zeiten der Sowjetunion dar. Die Streitkräfte Pekings stellen an Können jeden der „Schurkenstaaten“-Gegner in den Schatten, mit denen Washington in der unipolaren Ära konfrontiert war. Und in Anbetracht der Tatsache, dass Chinas Wirtschaftsleistung viel größer ist, als es die sowjetische je war, stellt China absehbar eine größere Herausforderung dar als die UdSSR.

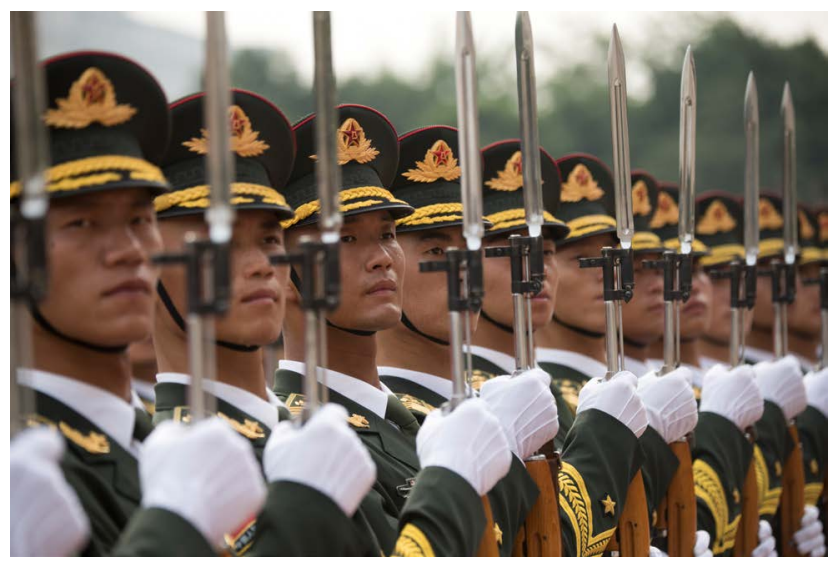

Abb. 1: Soldaten der chinesischen Volksbefreiungsarmee bei einem Paradeeinsatz

2 Kramp-Karrenbauer 2021.

3 Vgl. Internationaler Währungsfonds 2020.

4 U.S. Department of Defense 2019a, Dobbins/Shatz/Wyne 2018.
Folglich müssen die Vereinigten Staaten zum ersten Mal seit dem Kalten Krieg mit einem Gegner rechnen, der mit einer gewissen Wahrscheinlichkeit - selbst wenn Washington sich richtig ins Zeug legen würde - die Vereinigten Staaten in einem der wichtigsten Operationsgebiete der Welt besiegen könnte. Tatsächlich stellen einige glaubwürdige Beobachter und Verantwortliche die Frage, ob China nicht schon jetzt das militärische Gleichgewicht im Westpazifik zu seinen Gunsten verschoben hat. Das ist eine enorme Veränderung gegenüber jenen Tagen, als Washingtons Verteidigungsplaner es sich leisten konnten, darüber zu streiten, ob das US-Militär in der Lage sein sollte, mehreren Gegnern gleichzeitig oder lediglich nacheinander eine entscheidende Niederlage beizubringen und dann die Regierungen dieser Feinde zu stürzen. ${ }^{5}$

Wie aus den täglichen Schlagzeilen zu ersehen, ist China überdies in zunehmendem Maße bereit, seine neu gewonnene Macht selbstbewusst auszuspielen. Während China unter Deng Xiaoping eine Strategie des „Abwartens, bis seine Zeit gekommen ist“ verfolgte, hat Peking jetzt zweifelsohne diese Zurückhaltung abgelegt und durch Worte und Taten klargemacht, dass es seine immense Macht zu nutzen gedenkt, und dies auch in einer Weise, die zu Spannungen - oder Schlimmerem - mit anderen Staaten führt. Tatsächlich demonstriert Peking Tag für Tag, dass das alte Stereotyp zutrifft, wonach wachsende Macht, Stärke und Wohlstand zu Expansionsgelüsten führen. Die offizielle Einschätzung der US-Regierung ist daher, dass China zuerst nach regionaler Hegemonie in Asien streben wird und, sobald diese erreicht ist, nach einer globalen Vormachtstellung. ${ }^{6}$

Gleichzeitig sehen sich die Vereinigten Staaten und ihre vielen Verbündeten und Partner noch weiteren Herausforderungen gegenüber. Iran bedroht die Sicherheit Israels und der Golfstaaten im Nahen Osten, Nordkorea bedroht Südkorea und Japan in Asien, und der transnationale Terrorismus ist weiterhin eine ernstzunehmende Gefahr. Und selbstverständlich ist da noch Russland. Auch wenn die russische Wirtschaftsleistung viel geringer ist als die chinesische und die meisten Prognosen nahelegen, dass Russland bis zu einem gewissen Grad im Niedergang begriffen ist, bleiben einige unumstößliche Tatsachen bestehen: Russland investiert nach wie vor große Summen in sein Militär, es ist bereit, seine Streitkräfte und andere Instrumente nationaler Macht in einer Weise zu nutzen, die viele seiner Nachbarn (einschließlich vieler US-Verbündeter) als aggressiv betrachten, und die russi-

5 Shugart 2021.

6 U.S. Department of Defense 2019b. 
sche Bevölkerung scheint eine solche Strategie zu unterstützen und westlichem Druck standzuhalten. Die Regierung in Moskau, die offenkundig mit zentralen Elementen der internationalen Ordnung nach dem Ende des Kalten Krieges unzufrieden ist, verfügt dabei über Streitkräfte, die zwar kleiner sind als die Rote Armee, aber im Vergleich zu denen seiner Nachbarn, auch in Europa, sehr kampfstark. Es hat sein Militär in Georgien und auf der Krim direkt eingesetzt, getarnt und mit List in der Ukraine, und um Druck auf andere Staaten überall in seiner Peripherie auszuüben.

Aus der Sicht von Verteidigungsplanern bedeutet all dies, dass die Vereinigten Staaten mehrere potentielle militärische Szenarien auf drei verschiedenen, weit auseinanderliegenden Schauplätzen durchspielen müssen. In Asien sieht sich Washington der Herausforderung durch China gegenüber, insbesondere im Hinblick auf Taiwan, aber mit zunehmender Stärke der Volksbefreiungsarmee auch hinsichtlich anderer Verbündeter wie der Philippinen, Südkorea und Japan. Gleichzeitig haben sich die Vereinigten Staaten seit Langem darauf vorbereitet, bei der Verteidigung der Republik Korea gegen den Norden zu helfen, der nicht nur mit seinen Kernwaffen und Raketen eine Bedrohung darstellt, sondern auch über konventionelle Streitkräfte verfügt, deren Ausrüstung zwar veraltet ist, die aber sehr groß und gefährlich bleiben. In Europa bedeutet das Bekenntnis der USA zur NATO, dass die Vereinigten Staaten einen Angriff Moskaus auf ein NATO-Mitglied in Betracht ziehen müssen - am ehesten gegen vulnerable Verbündete im Osten, insbesondere die baltischen Staaten und Polen. Schließlich spielen die Vereinigten Staaten ungeachtet ihres Abzugs aus Afghanistan weiterhin eine sehr aktive militärische Rolle im Nahen Osten, etwa in Syrien, Irak und am Persischen Golf. Zudem haben sich die Vereinigten Staaten, insbesondere aus der Perspektive militärischer Planer, darauf konzentriert, wie man der Bedrohung entgegentreten kann, die der Iran für Verbündete und Partner der USA darstellt, insbesondere Israel. $^{7}$

Die Vereinigten Staaten werden unter den heutigen Bedingungen nicht mit all diesen potentiellen Konflikten im Wesentlichen aus eigener Kraft fertigwerden können, mit Sicherheit jedenfalls nicht, wenn diese gleichzeitig auftreten. Dies ist eine strukturelle Realität, die die Folge grundlegender Verschiebungen in der globalen Machtverteilung ist. Es ist nichts, was durch größere Entschlossen-

7 Zum Beispiel Wasser 2021, vgl. auch Joseph R. Biden, Jr., Interim National Security Strategic Guidance. White House, März 2021, 11, 15, https://www.whitehouse.gov/wp-content/uploads/2021/03/NSC-1v2. pdf. heit oder mit einer klugen Strategie überwunden werden könnte. Folglich können die Vereinigten Staaten diese Herausforderung nicht einfach durch stärkere Anstrengungen meistern - etwa durch eine drastische Steigerung ihrer Verteidigungsausgaben.

Sollten die Vereinigten Staaten ihre Verteidigungsausgaben erhöhen, könnte China entsprechend nachziehen, so dass dieser Schritt den USA nichts bringen würde. Vielleicht noch wichtiger ist die Tatsache, dass eine deutliche Erhöhung der US-Verteidigungsausgaben der amerikanischen Wirtschaft schaden könnte und so Sinn und Zweck dieser Maßnahmen konterkarieren würde. Zudem ist es höchst unwahrscheinlich, dass die Bevölkerung der Vereinigten Staaten solche drastischen Erhöhungen der Verteidigungsausgaben mitträgt. Die amerikanische Bevölkerung hat eher $\mathrm{zu}$ verstehen gegeben, dass sie gegen militärische Auslandseinsätze ist und keineswegs den Wunsch hat, mehr für das Militär auszugeben.

Man kann dieser veränderten Lage auch nicht durch eine „kluge“ oder „bessere“ Strategie beikommen. Kreative oder kluge Einsatzstrategien und -techniken können in bestimmten Situationen sehr nützlich sein - aber nur dann, wenn grundsätzlich eine militärische Machtbalance gegeben ist. Im Amerikanischen Bürgerkrieg konnten die rebellischen Südstaaten-Generäle eine Zeitlang ihre Gegenspieler aus den Nordstaaten überlisten, aber letztlich gab die Überlegenheit des Nordens den Ausschlag. Ebenso hatte Rommel dem Übergewicht alliierter Ressourcen in Nordafrika nichts entgegenzusetzen. Desgleichen wird keine noch so kluge Strategie eine allzu ungünstige militärische Balance im Fall Taiwan oder der baltischen Staaten wettmachen. Streitkräfte können zu einem bestimmten Zeitpunkt immer nur an einem Ort sein, und Bluffen wirkt nur begrenzt. Außerdem ist keineswegs ausgemacht, dass die Vereinigten Staaten bessere oder klügere Strategien als Peking oder Moskau haben werden. Fairerweise muss man sagen, dass es durchaus umgekehrt sein könnte. Jedenfalls gibt es keinen Grund dafür, anzunehmen, dass das Können immer auf unserer Seite sein wird.

\section{Konsequenzen für die US-Verteidigungspolitik}

Obgleich die politische Rhetorik hinterherhinkt, hat das US-amerikanische Verteidigungsestablishment dieses strukturelle Ungleichgewicht bereits erkannt. Tatsächlich haben die Vereinigten Staaten dies offiziell bereits mit ihrer National Defense Strategy (NDS) von 2018 getan, in der sie von ihrem Planungsstandard von zwei Kriegen 
(gleichzeitig) gegen „Schurkenstaaten,“ der in den Jahren nach dem Kalten Krieg bestimmend war, auf einen Standard umstellten, der sich auf das Gewinnen eines Krieges gegen eine Großmacht konzentrierte. Mit dieser Neuausrichtung gab Washington zu verstehen, dass die Vereinigten Staaten in ihren Verteidigungsvorbereitungen Plänen zur erfolgreichen Kriegsführung gegen eine Großmacht Priorität einräumen und nicht länger an der Zwei-KriegeStrategie der Vergangenheit festhalten würden. ${ }^{8}$ Und mit der NDS und anschließenden Erklärungen wurde deutlich, dass Washingtons Priorität in dieser Hinsicht China ist. ${ }^{9}$

Das bedeutet: die US-Verteidigungsplanung räumt in Asien China Priorität ein und will vor allem sicherstellen, dass US-Streitkräfte einen Krieg gegen China gewinnen können. Und es muss hinzugefügt werden, dass die Vereinigten Staaten noch keinesfalls davon überzeugt sind, dass sie die Fähigkeit besitzen, einen großen Krieg gegen China zu gewinnen. Tatsächlich sind die eigenen Erklärungen Washingtons über den Fortgang entsprechender Anstrengungen ernüchternd. Der US-Befehlshaber des Indo-Pacific Command hat im Frühjahr 2021 mit seiner ernüchternden, ja sogar beunruhigenden Einschätzung des militärischen Gleichgewichts im Westpazifik und dessen kontinuierlicher Verschlechterung große Aufmerksamkeit erregt. ${ }^{10}$ Entsprechend ist vollkommen klar, dass China in den kommenden Jahren einen wachsenden Anteil an den US-Verteidigungsanstrengungen beanspruchen wird.

Diese Entwicklung wird sehr unmittelbare, ja dramatische Folgen für die Sicherheit Europas haben. Es ist schlicht eine Tatsache, dass die Vereinigten Staaten weder die Macht noch die Mittel haben, China entgegenzutreten, wenn sie an ihrer überkommenen Verteidigungsstrategie festhalten. Auch wenn die USA, gemessen an den meisten Maßstäben, noch immer die stärkste Macht weltweit sind, sind sie einfach nicht stark genug, um an jener Verteidigungsstrategie festzuhalten, die sie verfolgten, als sie tatsächlich eine Monopolstellung innehatten. In praktischer Hinsicht verfügen die Vereinigten Staaten nicht über ein einsatzbereites Militär, das groß genug wäre, um zwei größere Kriege mit China und Russland gleichzeitig erfolgreich zu bestehen.

Dies bedeutet jedoch nicht unbedingt, dass sich die politischen Zusagen Washingtons verändern müssten. Möglicherweise kann Washington ungeachtet dieser Rea-

8 Mitre 2018.

9 Vgl. U.S. Department of Defense 2018, vgl. auch Lloyd J. Austin: Message to the Force - Memorandum for all Department of Defense Employees, 4. März 2021, S. 1.

10 Vgl. Davidson 2021 und Shugart 2021. lität das ganze Spektrum von Bündnis- und bündnisähnlichen Verpflichtungen aufrechterhalten. Aber anders als in den Jahren nach dem Ende des Kalten Krieges werden die USA gewiss nicht mehr in der Lage sein, dies weitgehend allein zu tun, mehrere Kriegsschauplätze weltweit gleichzeitig zu dominieren und auf jedem mehrere Rivalen zu bezwingen.

\section{Folgen für die Verteidigung und Sicherheit Europas}

Wie also sollten die Vereinigten Staaten mit dem Auseinanderklaffen zwischen ihren militärischen Fähigkeiten einerseits und dem Ausmaß der Herausforderungen für ihr Netzwerk von Verbündeten und Partnern andererseits umgehen?

Für Deutschland stellt sich in diesem Zusammenhang die Frage wie die USA dieses wachsende Ungleichgewicht beheben werden. Sowohl für die USA und, so sollte man jedenfalls meinen, auch für Deutschland wäre es ideal, wenn die Vereinigten Staaten diese Neuausrichtung im Einvernehmen mit ihren Alliierten durchführen könnten. Diese Herausforderung gemeinsam anzugehen, würde nicht bedeuten, einfach nur das zu tun, was im engeren Interesse der Vereinigten Staaten wäre. Die US-Verbündeten würden wohl kaum einer Strategie zustimmen, die von den Vereinigten Staaten diktiert oder die diese über Gebühr begünstigten würde. Das ist auch nicht das, was Washington anstrebt. Die Verteidigungsstrategie der USA in der Zeit nach dem Zweiten Weltkrieg (sowohl in ihren klügeren als auch in ihren draufgängerischen Momenten) war auf die Wahrung ganz und gar kollektiver Interessen ausgerichtet - die Sicherheit der Verbündeten und der entsprechenden regionalen Ordnungen, die diese Strukturen in Europa, Asien und dem Nahen Osten absichern. Schließlich berührt das, was in Osteuropa, am Persischen Golf oder auf der koreanischen Halbinsel passiert, wohl kaum US-amerikanische Kerninteressen. Es sind nur insofern amerikanische Interessen, als diese in Übereinstimmung mit den Interessen ihrer Alliierten und engen Partner definiert werden. ${ }^{11}$

Für Deutschland und andere US-Verbündete sind diejenigen unter diesen Interessen von besonderer Bedeutung, die ihre eigene Sicherheit betreffen, oder die ihrer Nachbarn sowie allgemein die Aufrechterhaltung fried-

11 Für eine Diskussion dieses Punkts im Rahmen der US-Bündnispolitik vgl. Mitchell 2021a. 
licher, stabiler und westlich orientierter regionaler Ordnungen. Dies soll nicht heißen, dass sich die Interessen der US-Verbündeten, selbst der mächtigsten wie Deutschland, perfekt mit denen der Vereinigten Staaten decken der mächtigsten Nation der Erde mit weltweiten Verpflichtungen und militärischen Stützpunkten. Vielmehr steht für US-Verbündete in ihren eigenen Regionen am meisten auf dem Spiel, und folglich ist ihre Entschlossenheit, zu handeln, sowie ihre Fähigkeit, dies zu tun, tendenziell dort am größten. Dies gilt für Deutschland genauso wie für die anderen Verbündeten und Partner der USA.

Vor diesem Hintergrund zeichnet sich von selbst eine Lösung für die Frage ab, wie sich in der gegenwärtigen Ära militärischer Herausforderungen die kollektive Verteidigung aufrechterhalten lässt. Die Vereinigten Staaten sollten ihre militärischen Anstrengungen auf die Bereiche konzentrieren, wo sie am nötigsten und folgenreichsten sind. Derweil sollten US-Verbündete und -Partner ihre eigenen Anstrengungen im Bereich Bündnisverteidigung gemäß einer bestimmten Logik verstärken: Sie sollten sich auf den Feldern mehr anstrengen, auf denen ihre Interessen direkt berührt sind und wo sie daher am ehesten nennenswerte militärische Beiträge leisten können. Diese Überlegungen führen zu einem Modell, in dem US-Verbündete und -Partner in Asien sich auf diese Region konzentrieren, während diejenigen im Nahen und Mittleren Osten ihr Augenmerk vor allem auf ihre Region richten. Europäischen Alliierten sollte mehr an ihrer eigenen Verteidigung und der Stabilität ihrer Nachbarregionen wie der Nordafrikas und des Mittelmeerraums gelegen sein. ${ }^{12}$ In diesem Kontext wären die Vereinigten Staaten am besten dran, wenn sie sich so weit wie möglich auf China und Asien konzentrieren und ihre militärischen Aktivitäten im Nahen Osten so weit wie möglich reduzieren würden. Gleichzeitig sollten sie ihnen wohlgesinnte Staaten in der Region, wie Israel und die Vereinigten Arabischen Emirate, befähigen, dort eine Führungsrolle zu übernehmen. Gleichzeitig kann und sollte Washington fest zu seinen NATO-Verpflichtungen stehen, aber zugleich müssen die europäischen Verbündeten jedoch stärkere Anstrengungen unternehmen und eine führende Rolle bei der konventionellen Verteidigung der Allianz akzeptieren.

Die Priorisierung Asiens durch Washington ist aus mehreren Gründen sinnvoll. Erstens ist die militärische Bedrohung durch China mit Abstand die akuteste Bedrohung für die US-amerikanische Bündnisarchitektur als Ganze. Das chinesische Bruttoinlandsprodukt ist weitaus größer als das Russlands, ganz zu schweigen von Iran,

12 Für eine ausführlichere Diskussion dieses Punktes vgl. Colby 2021. und Peking erhöht seine Verteidigungsausgaben weiterhin schnell und massiv. ${ }^{13}$ Auf absehbare Zeit sind die Vereinigten Staaten daher der einzige Staat, der in der Lage ist, China direkt entgegenzutreten. Andere Verbündete wie Japan, Südkorea, Taiwan und Australien können erhebliche Beiträge zur Verteidigung eines US-Verbündeten oder Quasi-Verbündeten wie Taiwan gegen China leisten, aber sie könnten niemals mit China in Asien fertigwerden, wenn die Vereinigten Staaten nicht die Führungsrolle übernehmen und den Hauptbeitrag leisten. Zweitens ist Asien der wichtigste Schauplatz der internationalen Politik. Dies hängt mit der Wirtschaftsleistung Asiens zusammen, die insgesamt sowohl die der Vereinigten Staaten als auch die Europas in den Schatten stellt - und wahrscheinlich im Lauf der Zeit größer sein wird als die beider zusammengenommen. ${ }^{14}$ China hegt hegemoniale Ambitionen in dieser so wichtigen Region und mit seiner wachsenden militärischen und wirtschaftlichen Stärke als bei weitem größte Macht Asiens hat es die Fähigkeit, diese Ziele zu erreichen, wenn ihm niemand entgegentritt. Und dies ist auch nicht nur für Asien ein Anlass zur Sorge. Auch wenn asiatische Staaten unmittelbar betroffen wären, sollte China die Vorherrschaft über die Region erringen, würden auch die Vereinigten Staaten und Europa schwer in Mitleidenschaft gezogen. Peking wäre dann in einer Position, die es ihm erlauben würde, seine Vormachtstellung zu nutzen, um einen beherrschenden Einfluss auf das wirtschaftliche, gesellschaftliche und politische Leben eines gespaltenen Europas und selbst der Vereinigten Staaten auszuüben. Es ist ein gemeinsames transatlantisches Interesse, dies zu verhindern. Folglich profitiert Europa selbst - indirekt, aber konkret - von der US-amerikanischen Fokussierung auf Asien.

Gleichzeitig hat der Aufstieg Chinas nicht die Bedrohung beseitigt, die Russland für die europäische NATO darstellt. Dadurch, dass der Aufstieg Chinas mehr Aufmerksamkeit und Ressourcen der USA beansprucht, könnte er dem Kreml Freiräume eröffnen. Dieser will offenbar die internationale Ordnung revidieren, wie sie sich nach dem Ende des Kalten Krieges herausgebildet hat und nutzt dabei seine militärische Überlegenheit dort, wo man

13 Daten laut Weltbank: BIP nach Kaufkraftparität (konstante USDollar von 2017) - China, Russische Föderation, Islamische Republik Iran; https://data.worldbank.org/indicator/NY.GDP.MKTP. PP.KD? end $=2019$ \&locations $=$ CN-RU-IR\&start $=1990 \&$ view $=$ chart Weltbank, letzter Zugriff 24. Mai 2021; vgl. auch CSIS China Power Project: What Does China Really Spend on its Military? China Power (CSIS), aktualisiert am 8. März 2021, https://chinapower.csis.org/ military-spending/.

14 Vgl. z. B. Valentina Romei/John Reed: The Asian Century Is Set to Begin, Financial Times, 26. März 2019. 
diese gegenüber den westlichen Verbündeten ausspielen kann. ${ }^{15}$ Das vordringlichste militärische Szenario für die NATO besteht heute darin, zu verhindern, dass Russland einen lokalen militärischen Vorsprung ausnutzt, um im Baltikum einzumarschieren oder Teile Polens zu besetzen, und so einen fait accompli zu schaffen. Russland würde dann versuchen, die übrigen NATO-Staaten davon $\mathrm{zu}$ überzeugen, dass es für sie zu kostspielig und im Vergleich zum möglichen Nutzen zu riskant wäre, diese neuen facts on the ground rückgängig zu machen. Um seine Gewinne zu sichern, könnte Moskau sein umfangreiches und sehr leistungsfähiges Nukleararsenal einsetzen, um massiv Druck auszuüben. ${ }^{16}$ Wenn Russland dabei erfolgreich wäre, hätte dies erhebliche Auswirkungen auf die Allianz. Es würde zumindest die Glaubwürdigkeit und den Zusammenhalt der Nordatlantischen Allianz untergraben, aber es könnte auch zu ihrer Schwächung oder sogar ihrem Zerfall führen. Und nur wenigen Ländern in Europa würde Instabilität in Osteuropa - oder, schlimmer noch, russische Vorherrschaft in diesem Gebiet - mehr schaden als Deutschland.

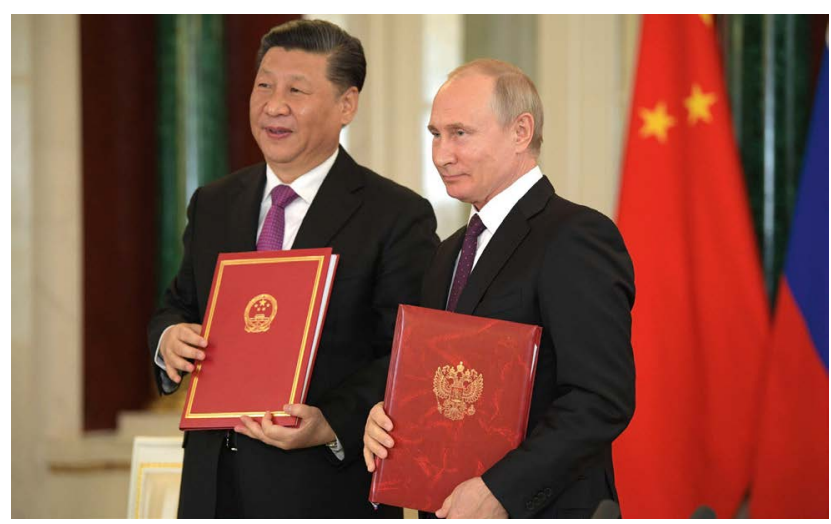

Abb. 2: Die Präsidenten Russlands und Chinas im Juni 2019

Da sowohl die Vereinigten Staaten als auch Europa von einem gut funktionierenden NATO-Bündnis mit starkem Zusammenhalt profitieren, haben die Vereinigten Staaten und ihre europäischen Verbündeten gemeinsam ein sehr großes Interesse daran, es nicht zu einem solchen Resultat kommen zu lassen. Nur das Bündnis trägt dazu bei, eine friedliche Stabilität auf dem europäischen Kontinent aufrechtzuerhalten. Aber aus den oben angeführten Gründen müssen die Vereinigten Staaten das Ausmaß der Beanspruchung ihrer militärischen Ressourcen aufgrund ihrer

15 Für eine eingehende Diskussion dieser bedeutenden Frage vgl. Mitchell 2021b.

16 Colby/Solomon 2015.
NATO-Beistandspflichten begrenzen, denn nur so können sie China in Asien wirkungsvoll entgegentreten.

\section{Die Europäer müssen mehr Ver- antwortung übernehmen}

Der mit Abstand beste Weg, um diese Lücke zu füllen, bestünde darin, dass die europäischen NATO-Staaten gemeinsam mit willigen Partnern wie Finnland und Schweden den Großteil der konventionellen Verteidigung NATO-Europas und Skandinaviens übernehmen. ${ }^{17}$ Die europäischen Verbündeten und Partner würden die Streitkräfte, die nötig wären, um einen russischen Einmarsch bei einem NATO-Alliierten oder einem skandinavischen Partner aufzuhalten oder idealerweise zurückzuschlagen, optimal ausbilden, einsatzbereit machen und dislozieren. Und im Konfliktfall wären sie darauf vorbereitet, dies mit amerikanischen Beiträgen zu tun, die im Vergleich $\mathrm{zu}$ denen, die gegenwärtig zu erwarten wären, begrenzt sein würden, da die USA vermeiden müssten, einen allzu großen Teil ihrer Streitkräfte und ihrer Aufmerksamkeit von Asien abzuziehen. Gleichzeitig könnten die Vereinigten Staaten weiterhin ihren NATO-Beistandspflichten nachkommen, sich an der Verteidigung jedes Bündnispartners zu beteiligen, aber sie würden ihre Beiträge weitgehend auf Streitkräfte konzentrieren, die sie bereitstellen könnten, ohne ihre Position im Pazifik allzu sehr zu schwächen, wie etwa schnell verlegbare Luftkampfmittel, fungible Weltraum- und Cyberfähigkeiten und selbstverständlich ihr nukleares Arsenal. Washington könnte auch selektiv in hochrentable Fähigkeiten für die europäische Verteidigung wie etwa Führungs-, Informations-, Computer-, Nachrichtengewinnungs-, Überwachungs- und Aufklärungsmittel (C4ISR) und fortgeschrittene Waffen investieren. ${ }^{18}$ Diese Strategie würde bei erfolgreicher Umsetzung die Verteidigung Europas und die Aufrechterhaltung einer starken transatlantischen Sicherheitspartnerschaft ermöglichen, während sie gleichzeitig der Tatsache gerecht würde, dass die Vereinigten Staaten China in Asien Priorität einräumen müssen.

Auch wenn diese Vorgehensweise eine einschneidende Veränderung bedeutete, sollte sie doch, allgemein betrachtet, voll und ganz mit den europäischen Kapazitäten übereinstimmen. Die Wirtschaftsleistung der NATO-

17 Brauss/Mölling 2021.

18 Für Beispiele solcher Investitionen vgl. Ochmanek/Wilson/Allen/ Meyers/Price 2017, S. 100. 
und der skandinavischen Staaten übertrifft bei weitem diejenige Russlands. Die meisten geben auch vergleichsweise wenig für Verteidigung aus, was dafür spricht, dass ihre Volkswirtschaften höhere Ausgaben verkraften könnten, um diesen Standard zu erreichen. ${ }^{19}$ In Anbetracht der Tatsache, dass die russischen Truppenverbände relativ klein sind, die in der Lage wären, eine Invasion der NATO durchzuführen, bedürfte es nur einer relativ geringen Aufstockung europäischer Streitkräfte, um abschreckend zu wirken und eine russische Invasion zu vereiteln. Dies gilt insbesondere dann, wenn die entsprechenden Kosten von einer breiten Gruppe europäischer Staaten getragen würden.

Gleichwohl würde unter diesen Umständen eine geschlossene und effektive Verteidigung der NATO enorm von einem einzelnen Akteur profitieren, der eine führende und besonders substantielle Rolle beim Schultern dieser Verteidigungslasten spielte. Wie bei jedem schwierigen gemeinschaftlichen Unterfangen ist ein starker und fähiger Anführer immer eine große Bereicherung. Ein solcher Akteur erlaubt kleineren und weniger mächtigen Akteuren, sich in einen bestehenden Ordnungsrahmen einzufügen, so dass sie gemeinsam leichter eine kritische Größe aufbauen können. Die Vereinigten Staaten haben in der NATO von jeher diese Rolle gespielt. Der einzige Staat in Europa, der in der nahen Zukunft einen beträchtlichen Teil dieser Führungsrolle übernehmen könnte, wäre Deutschland. Allein Deutschland hat die Bevölkerung, die wirtschaftliche Potenz und den hohen Entwicklungsstand, die politischen Beziehungen und die geografische Lage im Zentrum Europas, um diese Rolle einzunehmen. Nur Großbritannien und Frankreich kommen Deutschland in Bezug auf wirtschaftliche und latente militärische Macht in Europa annähernd nahe, aber beide haben eine deutlich kleinere Bevölkerung. Außerdem sind beide auch geografisch weiter von dem wichtigsten potentiellen Gefahrengebiet im Osten der NATO entfernt. Großbritannien hat mittlerweile die Europäische Union verlassen, was seine Fähigkeit erschwert, eine solche Führungsrolle zu übernehmen. Frankreich fehlt die wirtschaftliche Basis, um eine solche Führungsposition anzustreben. Überdies hat Paris starke Interessen im Mittelmeerraum und in Nordafrika, die einen erheblichen Teil seiner Aufmerksamkeit und seiner militärischen Fähigkeiten in Anspruch nehmen.

Von daher ist Deutschland der natürliche und tatsächlich der einzige wirkliche Kandidat, um als der füh-

19 Daten gemäß der SIPRI Military Expenditure Database auf der Webseite des Stockholm International Peace Institute, https://www. sipri.org/databases/milex. rende Rahmenstaat für die Neuaufstellung der europäischen Verteidigung zu dienen. Am reibungslosesten wäre es, wenn Deutschland eine deutlich maßgeblichere Führungsrolle für die konventionelle Verteidigung Europas innerhalb der NATO und gegebenenfalls der Europäischen Union übernehmen würde. Dabei würde sich Berlin streng darauf konzentrieren, die Boden-, taktischen Luft-, Logistik- und zentralen High-Tech-Fähigkeiten bereitzustellen, die für eine Verteidigung der östlichen NATO-Staaten und Skandinaviens benötigt würden. Die Verteidigung des Baltikums, Polens und/oder Finnlands wären die zentralen Planungsszenarien. Eine deutsche Kernstreitmacht würde anderen Staaten erlauben, ihre Streitkräfte in eine schlagkräftige Gesamtheit zu integrieren. Frontstaaten im Osten wie Polen und Finnland sowie Schweden könnten sich auf die direkte Verteidigung ihres Territoriums oder die ihrer bedrohten unmittelbaren Nachbarn konzentrieren, während andere europäische Staaten wie Großbritannien, Frankreich, Dänemark, die Niederlande sowie Kanada ihre Streitkräfte in dem Maße einbringen könnten, wie es für die kollektiven Verteidigungsanstrengungen von Nutzen wäre. Derweil könnten sich die Vereinigten Staaten darauf konzentrieren, Fähigkeiten mit hohem Mehrwert bereitzustellen. Diese wären ein wichtiger Beitrag für den Kampf in Europa, ohne dass die USA allzu viele Ressourcen von ihrer Priorität, Asien, abziehen müssten.

\section{Die besondere Rolle Deutschlands}

Aber ist das ein plausibles Szenario? Es mag sich theoretisch überzeugend anhören, aber ist es auch realistisch, insbesondere in einem Deutschland, welches derzeit die entsprechende Erhöhung seiner Verteidigungsausgaben oder die Übernahme mehr sicherheitsbezogener Verantwortlichkeiten zu vermeiden sucht? Trotz der offenkundigen Schwierigkeiten, ist es zu einem gewissen Grade plausibel anzunehmen, dass Deutschland sich dafür entscheiden könnte - aus mehreren Gründen:

Erstens stünde ein solches Vorgehen im Einklang mit den erklärten außenpolitischen Zielen Deutschlands. Kein Land lobt den Multilateralismus, gemeinsame Anstrengungen und die europäische Zusammenarbeit beredter und beharrlicher. Was könnte Deutschlands Bekenntnis zu diesen edlen Zielen besser zum Ausdruck bringen als die Übernahme einer Führungsverantwortung innerhalb der NATO, wenn diese Anstrengung besonders dringend benötigt wird? 
Zweitens ist es in Deutschlands höchstem Interesse, dafür zu sorgen, dass seine Nachbarschaft stabil und freundlich bleibt. Wenn Deutschland diese Rolle nicht übernimmt, erhöht Berlin das Risiko von Instabilität und sogar direkter russischer Aggression in seiner unmittelbaren Nachbarschaft. Dies würde zweifellos die Sicherheit Deutschlands und wahrscheinlich auch seine wirtschaftliche Situation verschlechtern.

Drittens sollte ein solches Vorgehen auch mit der soliden Finanzpolitik in Deutschland in Einklang stehen, insbesondere angesichts der Tatsache, dass sich Berlin bereits verpflichtet hat, seine Verteidigungsausgaben $\mathrm{zu}$ erhöhen, um seine beim NATO-Gipfel in Wales gemachte Zusage einzuhalten, zwei Prozent seines BIP für Verteidigung auszugeben. ${ }^{20}$ Für Deutschland geht es im Wesentlichen darum, die notwendigen Mittel aufzubringen, um den kritischen Kern kampffähiger Verbände zu stellen, die zur Abschreckung und Verteidigung der baltischen Staaten notwendig sind. Es geht nicht darum, eine massive Aufrüstung in die Wege zu leiten. Außerdem würde Deutschland dies nicht allein tun; vielmehr geben europäische Länder wie Großbritannien, Polen, Schweden und einige andere bereits beträchtliche Summen für Verteidigung aus, die allerdings in einem Rahmenwerk unter deutscher Führung effizienter verwendet werden könnten als es derzeit der Fall ist. ${ }^{21}$ Außerdem ist, wie schon erwähnt, die Bedrohung aus Russland zwar in mancher Hinsicht sehr ernst, aber sie bleibt doch begrenzt. Die russische Wirtschaft ist schon viel kleiner als die deutsche Wirtschaft für sich genommen, ganz zu schweigen vom Bruttoinlandsprodukt aller europäischen NATO-Staaten zusammengenommen. Demgemäß sollten deutliche, aber keineswegs dramatische Steigerungen der deutschen Verteidigungsausgaben, in Verbindung mit denjenigen anderer europäischer Verbündeter und Partner eine wirkungsvolle konventionelle Fähigkeit zur Abschreckung und Verteidigung für die europäische NATO bewirken.

Viertens stünde eine deutsche Verantwortungsübernahme voll und ganz in Einklang mit dem Nachkriegsvermächtnis Deutschlands. Ein solches Vorgehen würde sich auf die Bündnisverteidigung konzentrieren und wäre in alliierte und europäische Strukturen eingebunden. Damit wäre Deutschland weit entfernt von dem aggressiven, uni-

20 Staats- und Regierungschefs, die an dem Treffen des Nordatlantikrats in Wales teilgenommen haben, "Wales Summit Declaration," North Atlantic Treaty Organization, 5. September 2014, https://www. nato.int/cps/en/natohq/official_texts_112964.htm.

21 Daten gemäß der SIPRI Military Expenditure Database auf der Webseite des Stockholm International Peace Institute, https://www. sipri.org/databases/milex. lateralen Streben nach Vorherrschaft, die Deutschlands Nachbarn aus historischen Gründen vor 1945 von Berlin fürchteten. Die Streitkräfte, die ein solches Deutschland aufbauen und einsetzen würde, würden sich auf konventionelle Bündnisverteidigung, nicht auf Machtprojektion, geschweige denn Aggressionen konzentrieren. Was die praktische Planung anlangt, würde Deutschland sein Militär sehr spezifisch zur Verteidigung der östlichen NATO-Staaten und seiner skandinavischen Nachbarn entwickeln und stationieren. Damit würden glaubwürdige, konkrete Beweise für die wohlwollende, rein defensive Natur der Absichten erbracht, die Deutschland mit seiner Aufrüstung verfolgt. Sollten Deutschlands Nachbarn in Sorge sein wegen des potentiellen Missbrauchs dieser Streitkräfte durch Berlin, so könnten sie sicherstellen, dass kritische Teile einer solchen kollektiven Streitmacht nicht durch die Bundeswehr gestellt werden. Dadurch können die Missbrauchsmöglichkeiten, so unwahrscheinlich sie auch sein mögen, zusätzlich begrenzt werden.

Tatsächlich wäre dieses Vorgehen eher eine Erfüllung als eine Zurückweisung der Nachkriegsrolle Deutschlands und der des westlichen Bündnisses allgemein. Die zentrale Lehre, die die Westmächte aus dem Zweiten Weltkrieg zogen, war nicht, dass es ratsam wäre, abzurüsten, sondern, dass es einer entschlossenen kollektiven Verteidigung bedürfe: Freie Länder müssten bereit und in der Lage sein, nicht nur sich selbst, sondern auch einander zu verteidigen. Nur so können sie verhindern, dass aggressive, autoritäre Staaten sie der Reihe nach auseinandernehmen. Obwohl der Krieg gerade erst zehn Jahre zurücklag, unterstützten die Westmächte die robuste Wiederbewaffnung der Bundesrepublik nach 1955 als Teil der alliierten Verteidigungsanstrengungen, da die Größe und die Schlüssellage Westdeutschlands bedeuteten, dass es ein zentraler Akteur der westlichen Bündnisverteidigung sein musste.

Ungeachtet der düsteren Militärgeschichte Deutschlands war einer der nobelsten Beiträge der Bundesrepublik in der Nachkriegszeit just militärischer Natur: seine bedeutenden Beiträge zur konventionellen Verteidigung der NATO während der verbleibenden Dekaden des Kalten Kriegs. Tatsächlich profitierte kein Land mehr von dem Bekenntnis der NATO zur Bündnisverteidigung als die Bundesrepublik selbst: zunächst bei der Verteidigung Westdeutschlands, das direkt an der Front zum Warschauer Pakt lag, und dann dadurch, dass die NATO den günstigen Ausgang des Kalten Krieges und die Wiedervereinigung des Landes unter einer freien Regierung ermöglichte. Wie könnte Deutschland besser zeigen, dass es die richtigen Lehren aus seiner Vergangenheit gezogen hat und der NATO dankbar ist, als dadurch, dass es den 
Ländern östlich von ihm, die während des 20. Jahrhunderts so viel Leid durchmachten, ein ähnliches Niveau konventioneller kollektiver Verteidigung anbietet?

\section{Die Alternativen}

Der letzte Grund, wieso Deutschland geneigt sein dürfte, eine führende Rolle bei der Verteidigung Europas zu spielen, ergibt sich aus der Betrachtung der wahrscheinlichen Alternative. Kurz gesagt: Wenn Deutschland bei der konventionellen Verteidigung Europas keine derartige Führungsrolle übernimmt, hätte dies wahrscheinlich ein Sicherheitsvakuum in der unmittelbaren Nachbarschaft Deutschlands zur Folge, denn Amerika wird wohl kaum weiterhin die Lasten für die Verteidigung der Nachbarn Deutschlands tragen. Wenn Deutschland sich gegen eine solche Rolle entscheidet, zwingt es den Vereinigten Staaten daher faktisch eine Entscheidung auf: Entweder sie versuchen, Abschreckung und Verteidigung in Europa gegen Russland aufrechtzuerhalten, und zwar auf Kosten ihrer Fähigkeit, dies in Asien gegen China zu tun, oder sie verstärken den Fokus auf Asien, was das Risiko einer russischen Aggression in Europa erhöht. Wir wissen nicht, wie Washington sich in einem solchen Fall verhalten wird. Es ist anzunehmen, dass die Vereinigten Staaten versuchen werden, eine strikte Entweder-oder-Entscheidung zu vermeiden. Aber wenn es zu einer Situation kommen sollte, in der Washington eine solche Entscheidung treffen müsste, dann würde es Asien den Vorrang geben und Risiken in Europa akzeptieren.

Diese Feststellung ist die schlichte Folgerung aus der weiter oben dargelegten Logik. Asien ist in Anbetracht der überragenden Größe seines Marktes die bedeutendste Region der Welt, und China - die zweite globale Supermacht, auf die ungefähr die Hälfte des Bruttosozialprodukts Asiens entfällt - ist ein plausibler Anwärter auf eine regionale Vormachtstellung. Dies hätte gravierende negative Folgen für den Wohlstand, die Freiheit und letztlich die Sicherheit der Amerikaner. China hat derzeit sowohl die Macht als auch offensichtlich die Entschlossenheit, diesen Weg zu gehen und eine beherrschende Stellung in Asien - dem größten Markt der Welt - zu erlangen.

Europa wiederum ist zwar ein bedeutender Markt, aber seine Wirtschaftsleistung ist deutlich geringer als diejenige Asiens. Russland ist zwar gefährlich und militärisch leistungsfähig, aber eine viel unbedeutendere Macht als China. In Anbetracht der Tatsache, dass Russland ein kleineres Bruttoinlandsprodukt hat als Deutschland und eine Reihe anderer europäischer Staaten, hat es keine realistischen Aussichten darauf, das Ausmaß an Vorherrschaft über Europa zu erreichen, welches China über Asien anstrebt. Wenn Russland gewaltsam gegen östliche NATO-Staaten vorgehen würde, würde dies wohl entschlossene Gegenmaßnahmen durch Staaten wie Deutschland, Frankreich und Großbritannien auslösen. Während also einige in Moskau danach streben mögen, die Macht zurückzuerlangen, die der Kreml während des Kalten Kriegs besaß, ist dies in absehbarer Zukunft keine realistische Aspiration.

Selbstverständlich wäre es den Vereinigten Staaten entschieden lieber, nicht vor die Wahl gestellt zu werden, zwischen Europa und Asien entscheiden zu müssen. Aber solange das Zaudern Deutschlands anhält, bleibt dieses Szenario auf dem Tisch und Washington müsste sich für eines der beiden Ergebnisse entscheiden. Das eine Asien unter Vorherrschaft Chinas - hätte viel gravierendere Folgen als das andere - wenn Russland freie Hand für Angriffe auf osteuropäische Länder hätte. Vor die Wahl gestellt, wäre es für Washington klüger, Asien Priorität einzuräumen und das Risiko in Europa zu akzeptieren.

Dieses Dilemma ist schon näher, als die meisten glauben. In Anbetracht der Tatsache, dass sich Washington schon jetzt besorgt fragt, wie erfolgreich es seine Verbündeten und Taiwan im Pazifik verteidigen könnte, kann man es nicht mehr als ein „langfristiges“ Problem ansehen, das weit hinter dem Horizont liegt. Vielmehr ist es schon jetzt da. Schon jetzt müssten sich die Vereinigten Staaten den Kopf darüber zerbrechen, wie sehr ein starkes Engagement bei einem Konflikt in Europa die US-Verteidigungskapazitäten in Asien schwächen würde. Die Vereinigten Staaten hatten bereits Probleme mit dem Mangel an moderner Munition, und sie haben nur sehr wenige schwere Bomber, die tief in den gegnerischen Luftraum eindringen können. Sie haben eine ausgedehnte Logistik-Infrastruktur und ihre U-Boote leiden gerade an einer „Welle von Ausfällen.“222 Zudem können kritische US-Streitkräfte nicht an zwei Orten gleichzeitig eingesetzt werden, und viele, insbesondere entscheidende Seestreitkräfte und Logistik-Unterstützungskräfte bräuchten Wochen, wenn nicht länger, um zwischen den Kriegsschauplätzen Europa und Westpazifik zu pendeln. ${ }^{23}$

Den Europäern sollte dabei eines klar sein: Angesichts der Tatsache, dass die USA China in Asien Priorität einräumen, werden die Vereinigten Staaten selbst dann, wenn ein Krieg in Europa ausbrechen sollte, nicht zu große Risiken

22 Vgl. Govini: The 2020 Federal Scorecard, 9, https://www.govini. com/wp-content/uploads/2021/04/Govini-2020-Federal-Score card-1.pdf, vgl. auch Richard 2021 und Walton/Boone/Schramm 2019. 23 Walton/Boone/Schramm 2019, $11 \mathrm{f}$. 
in Asien eingehen und dadurch opportunistischen Angriffen Chinas Tür und Tor öffnen. Dies bedeutet, praktisch gesehen, dass die USA ihr Engagement in einem europäischen Krieg begrenzen müssten. Dies dürfte eine erhebliche und wahrscheinlich wachsende Lücke in der Verteidigung Europas nach sich ziehen. Daraus folgt: Wenn die europäischen Verbündeten und insbesondere Deutschland sich weiterhin weigern, ihre Verteidigungsanstrengungen zu erhöhen, werden die Vereinigten Staaten ihre Aufmerksamkeit nicht von Asien abwenden oder ihre Verteidigungsanstrengungen erhöhen, um dieses Vakuum zu füllen. Vielmehr werden sie sich auf Asien konzentrieren und die Risiken akzeptieren, die eine solche Weigerung der Europäer in Europa mit sich bringt.

Es ist wichtig zu betonen, dass diese Überlegungen einer strategischen Logik folgen und dass es nicht um Verstimmungen geht. Vor diese, hier ausgeführte unangenehme Wahl gestellt, bestünde der vernünftigste strategische Kurs für die Vereinigten Staaten darin, sich für Asien zu entscheiden. Aber es wäre auch ethisch gerechtfertigt und daher wahrscheinlich politisch vertretbar und vielleicht sogar in den Vereinigten Staaten populär, diese Politik zu verfolgen. Wenn sich die Europäer weigern, ihre Verteidigungsanstrengungen $\mathrm{zu}$ erhöhen, um sich vor dem Hintergrund weitaus gravierender Herausforderungen in Asien selbst zu verteidigen, wäre es für Amerika kaum sinnvoll, sich noch größere Lasten aufzuerlegen, um europäische Defizite zu kompensieren. Die Tatsache, dass die Amerikaner zunehmend China als größte Bedrohung ansehen, spricht in Verbindung mit dem erklärten Willen auf beiden Seiten des politischen Spektrums, Auslandseinsätze zu reduzieren, dafür, dass die Priorisierung Asiens breite politische Unterstützung seitens des amerikanischen Volkes erhalten würde.

\section{Ausblick}

Selbstverständlich wäre dieses Ergebnis schlecht für die Europäer und die Amerikaner. Niemand hätte etwas davon, wenn Europa russischen Aggressionen vielfältige Angriffsflächen bieten würde. Aber wenn Amerika vor der Alternative steht, muss es Asien den Vorrang geben. Es ist wichtig, dass die Europäer, vor allem aber die Deutschen, diesen Punkt genau verstehen, weil es heute die Neigung gibt, die Debatte über Lastenteilung so aufzufassen, als wäre sie in erster Linie von persönlichen Temperamenten, Gefühlen und Fragen der Fairness bestimmt. Diese spielen dabei selbstverständlich eine Rolle, aber die grundlegenden Faktoren sind sehr real und viel tiefgreifender.
Tatsache ist, dass die Vereinigten Staaten nicht länger die einzige Supermacht der Welt sind - auch China ist eine, und es steht zu erwarten, dass es zur Vormacht in Asien wird, wenn es nicht gebremst wird. Wenn China Asien dominiert, wird es eine beherrschende wirtschaftliche und geopolitische Stellung in der Welt erlangen, die es ihm erlauben wird, überall einen bestimmenden Einfluss auszuüben. Washington kann es sich daher nicht leisten, auf diesem entscheidenden Schauplatz, Asien, beim militärischen Gleichgewicht Fehler zu machen. Daher haben die strategischen Interessen der USA eindeutig Vorrang. Aber dies ist auch im europäischen Interesse. Ein solches hegemoniales China mag eine Bedrohung für die Vereinigten Staaten darstellen, die eine kohäsive Supermacht sind. Für ein gespaltenes, uneiniges Europa wäre es noch eine viel größere Bedrohung. Von einer hegemonialen Position in Asien aus wäre Peking zweifellos in der Lage, auch einen beherrschenden Einfluss auf Europa auszuüben, die Terms of Trade zu diktieren, wirtschaftliche Aktivitäten zu seinen Gunsten zu steuern und sich in die Innen- und Außenpolitik von Ländern einzumischen, um nur einige wenige wahrscheinliche Ergebnisse zu benennen, die man schon heute sehen kann. Tatsächlich versucht China dies bereits bei vielen europäischen Ländern. Ein hegemoniales Peking könnte seine Ziele noch leichter erreichen, und es hätte keinen Grund, sie aufzugeben. Etwas vereinfacht ausgedrückt: Wenn Peking erst einmal Asien beherrscht, wird es früher oder später auch einen viel größeren Einfluss auf Europa ausüben.

Diese Herausforderung lässt sich am besten durch transatlantische Kooperation und Koordinierung unserer komplementären Anstrengungen bewältigen. ${ }^{24}$ Und es gibt einen erfolgversprechenden, vernünftigen Weg, dies zu tun: die Vereinigten Staaten konzentrieren sich in Asien auf China, aber sie stehen weiterhin treu zur NATO, während europäische Staaten unter Führung Deutschlands die Hauptrolle bei der konventionellen Verteidigung Europas gegen Russland übernehmen. Europäer und vor allem Deutsche sollten die Folgen verstehen, die Tatenlosigkeit haben wird. Die jetzige amerikanische Regierung mag ihren europäischen Partnern versichern, dass das Bekenntnis der USA zur NATO unerschütterlich und heilig ist, aber diese Hymnen werden sich als bedeutungslos erweisen, wenn sich die Allianz nicht anpasst.

24 Vgl. NATO Reflection Group 2020 und de Maiziere/Mitchell 2021. 


\section{Literatur}

Bagger, Thomas (2019): The World According to Germany: Reassessing 1989, The Washington Quarterly, 41 (4), 53-63

Brauss, Heinrich/Mölling Christian (2021): NATO 2030 - The Military Dimension. Rom: NATO Defense College, 21. April 2021. https:// www.ndc.nato.int/news/news.php?icode $=1551$

Colby, Elbridge (2021): Allied Interests, The National Interest. No. 173, Mai/Juni 2021, 35-45, https://nationalinterest.org/ issue/mayjun-2021

Colby, Elbridge/Solomon, Jonathan (2015): Facing Russia: Conventional Defence and Deterrence in Europe, Survival, 57 (6), S. 21-50

De Maizière, Thomas/Mitchell, A. Wess (2021): NATO Needs to Deal with China Head-On, Foreign Policy, 23. Februar 2021, https:// foreignpolicy.com/2021/02/23/nato-china-brussels-summitbiden-europe-alliance/

Dobbins, James/Shatz, Howard J./Wyne, Ali (2018): Russia is a Rogue, not a Peer; China is a Peer, Not a Rogue. Santa Monica, CA: The RAND Corporation

Kramp-Karrenbauer, Annegret (2021): This is a Huge Break with the Past, Internationale Politik Quarterly, 28. April 2021, https:// ip-quarterly.com/en/huge-break-past

Mitchell, A. Wess (2021a): The Case for Renovating American Alliances. Forum for American Leadership, Edited Volume (in Kürze erscheinend).

Mitchell, A. Wess (2021b): A Sequencing Strategy to Handle America's Simultaneity Problem, The National Interest, Sommer/Herbst-Ausgabe, 2021 (in Kürze erscheinend).

Mitre, Jim (2018): A Eulogy for the Two-War Construct, The Washington Quarterly, 41 (4), S. 7-30

Ochmanek, David/Wilson, Peter A./Allen, Brenna/Meyers, John Speed/Price, Carter C. (2017): U.S. Military Capabilities and Force for a Dangerous World: Rethinking the U.S. Approach to Force Planning. Santa Monica, CA: The RAND Corporation

Shugart, Thomas H. III (2021): Trends, Timelines and Uncertainty: An Assessment of the Military Balance in the Indo-Pacific. Testimony before the Senate Foreign Relations Committee hearing on Advancing Effective U.S. Policy for Strategic Competition with China in the Twenty-First Century, 17. März 2021, https://www.foreign.senate.gov/imo/media/ doc/031721_Shugart_Testimony.pdf

Walton, Timothy A./Boone, Ryan/Schramm, Harrison (2019): Sustaining the Fight: Resilient Maritime Logistics for a New Era. Washington, DC: Center for Strategic and Budgetary Assessments; https://csbaonline.org/uploads/documents/ Resilient_Maritime_Logistics.pdf.

Wasser, Becca (2021): Drawing Down the U.S. Military Responsibly, in: Frederic Wehry/Michelle Dunne (eds.): From Hardware to Holism: Rebalancing America's Security Engagement with Arab States. Washington, DC: Carnegie Endowment for International Peace, https://carnegieendowment.org/2021/05/18/drawingdown-u.s.-military-responsibly-pub-84527, Kapitel 3

\section{Amtliche Dokumente und Erklärungen}

Davidson, Phillip S. (2021): Indo-Pacific Command Posture. Statement by the US Navy Commander of the U.S. Indo-Pacific Command before the Senate Armed Services Committee, 9. März 2021, https://www.armed-services.senate.gov/imo/ media/doc/Davidson_03-09-21.pdf

International Monetary Fund (2020): World Economic Outlook Database: Report for Selected Countries and Subjects, https:// www.imf.org/en/Publications/WEO/weo-database/2020/ October/weo-report?; letzter Zugriff 25. Mai 2021

NATO Reflection Group (2020): NATO 2030: United for a New Era. Brussels: NATO, https://www.nato.int/nato_static_fl2014/ assets/pdf/2020/12/pdf/201201-Reflection-Group-FinalReport-Uni.pdf

Richard, Charles A. (2021): Statement of the Commander of the United States Strategic Command before the Senate Armed Services Committee, 20. April 2021, S. 15, 17, https://www.armed-services.senate.gov/imo/media/doc/ Richard04.20.2021.pdf

U.S. Department of Defense (2018): Summary of the 2018 National Defense Strategy of the United States of America: Sharpening the American Military's Competitive Edge. Washington, DC: U.S. Department of Defense, https://dod.defense.gov/ Portals/1/Documents/pubs/2018-National-Defense-StrategySummary.pdf

U.S. Department of Defense (2019a): Annual Report to Congress: Military and Security Developments Involving the People's Republic of China. Washington, DC: U.S. Department of Defense

U.S. Department of Defense (2019b): Indo-Pacific Strategy Report: Preparedness, Partnerships, and Promoting A Networked Region. Washington, DC: U.S. Department of Defense 